\title{
Design of a Lambda system for population transfer in superconducting nanocircuits
}

\author{
G. Falci, ${ }^{1,2,3, *}$ A. La Cognata, ${ }^{3}$ M. Berritta,,${ }^{1, \dagger}$ A. D’Arrigo, ${ }^{2}$ E. Paladino,,${ }^{1,2,3}$ and B. Spagnolo ${ }^{4}$ \\ ${ }^{1}$ Dipartimento di Fisica e Astronomia, Università di Catania, Via Santa Sofia 64, 95123 Catania, Italy \\ ${ }^{2}$ CNR-IMM UOS Università (MATIS), Consiglio Nazionale delle Ricerche, Via Santa Sofia 64, 95123 Catania, Italy \\ ${ }^{3}$ Centro Siciliano di Fisica Nucleare e Struttura della Materia, Via Santa Sofia 64, 95123 Catania, Italy \\ ${ }^{4}$ Dipartimento di Fisica e Chimica, Università di Palermo, Group of Interdisciplinary Physics and CNISM, \\ Unità di Palermo, Viale delle Scienze, Ed.18, I-90128 Palermo, Italy \\ (Received 25 February 2013; revised manuscript received 9 May 2013; published 20 June 2013)
}

\begin{abstract}
The implementation of a Lambda scheme in superconducting artificial atoms could allow detection of stimulated Raman adiabatic passage (STIRAP) and other quantum manipulations in the microwave regime. However, symmetries which on one hand protect the system against decoherence yield selection rules which may cancel coupling to the pump external drive. The tradeoff between efficient coupling and decoherence due to broad-band colored noise $(\mathrm{BBCN})$, which is often the main source of decoherence, is addressed in the class of nanodevices based on the Cooper pair box (CPB) design. We study transfer efficiency by STIRAP, showing that substantial efficiency is achieved for off-symmetric bias only in the charge-phase regime. We find a number of results uniquely due to non-Markovianity of BBCN, namely (a) the efficiency for STIRAP depends essentially on noise channels in the trapped subspace; (b) low-frequency fluctuations can be analyzed and represented as fictitious correlated fluctuations of the detunings of the external drives; and (c) a simple figure of merit for design and operating prescriptions allowing the observation of STIRAP is proposed. The emerging physical picture also applies to other classes of coherent nanodevices subject to BBCN.
\end{abstract}

\section{INTRODUCTION}

The rapid technological progress in quantum-state engineering in superconducting nanodevices demands the implementation of new advanced techniques of quantum control. Stimulated Raman adiabatic passage (STIRAP) ${ }^{1,2}$ is a powerful method in quantum optics, which is still largely unexplored in the solid-state realm. Using ac driving fields in $\Lambda$ configuration [see Fig. 1(a)] a quantum $M>2$ state system is trapped into a subspace spanned by the two longest lived states. Control in this trapping subspace can be achieved by adiabatic time evolution induced by properly crafted pulses, allowing for instance to prepare a given target state. ${ }^{3,4}$ Adiabatic passage used in STIRAP guarantees highly efficient and selective population transfer in atomic and molecular systems. ${ }^{1,2}$

In the last few years it has been proposed that multilevel quantum coherent effects ${ }^{4}$ could be observed in superconducting nanodevices, for instance electromagnetically induced transparency $(\mathrm{EIT})^{5}$ and selective population transfer by STIRAP $^{6-9}$ or related protocols. ${ }^{10}$ This would be important both from a fundamental point of view, since coherent dynamics in multilevel atoms clearly displays beautiful interference phenomena, ${ }^{4}$ and for applications. These include the implementation of microwave quantum switches, ${ }^{11}$ the manipulation of solid-state qubit circuits ${ }^{10,12}$ and the fascinating perspectives of coupling strongly such nanodevices to electromagnetic ${ }^{13}$ or nanomechanical quantized modes. ${ }^{9}$ Many applications are discussed in the recent review Ref. 14. Very recently a few experiments have demonstrated features of multilevel coherence in such devices, such as the Autler-Townes (AT) splitting, ${ }^{15,16}$ EIT, $^{17}$ preparation and measurement of threestate superpositions, ${ }^{18}$ dynamical AT control ${ }^{11}$ and coherent population trapping. ${ }^{19}$
In all the above experiments, except that of Ref. 19, the multilevel system was driven in the ladder configuration. ${ }^{4}$ Indeed in order to implement a $\Lambda$ configuration the device Hamiltonian should be strongly asymmetric, which may be achieved by a proper external biasing, ${ }^{7-9,14}$ otherwise selection rules prevent driving efficiently the pump transition. However, the longest decoherence times in quantum bits are achieved by biasing the devices at (or near) parity symmetry points. Hence difficulties in implementing a $\Lambda$ configuration in superconducting nanodevices raise a fundamental design issue. In particular, low-frequency noise, which is known to determine the performances of systems operated as quantum bits $^{20}$ and which is minimized at symmetry points, is shown in this work to play a major role in degradation of efficiency in STIRAP. So far the effect of decoherence in multilevel superconducting artificial atoms has been addressed using Markovian master equations. In this work we address decoherence effects due to a solid-state environment where a strong non-Markovian noise component is also present. From the exquisite sensitivity of coherence to operating conditions, and to design parameters of the device, we determine the prescriptions for the demonstration of a $\Lambda$ scheme in realistic superconducting nanocircuits.

We tackle this problem by a quantitative analysis of a class of superconducting nanocircuits, namely those based on the Cooper pair box ${ }^{21}$ (CPB, see Fig. 4). This is an important case-study encompassing several different coherent nanodevices which have already successfully implemented quantum bits. $^{22-26}$ The emerging physical picture is even more general, holding true for nanodevices suffering mainly from the presence of low-frequency noise.

The main message of this work is twofold. First we find that observation of STIRAP should be possible with devices 
(a)
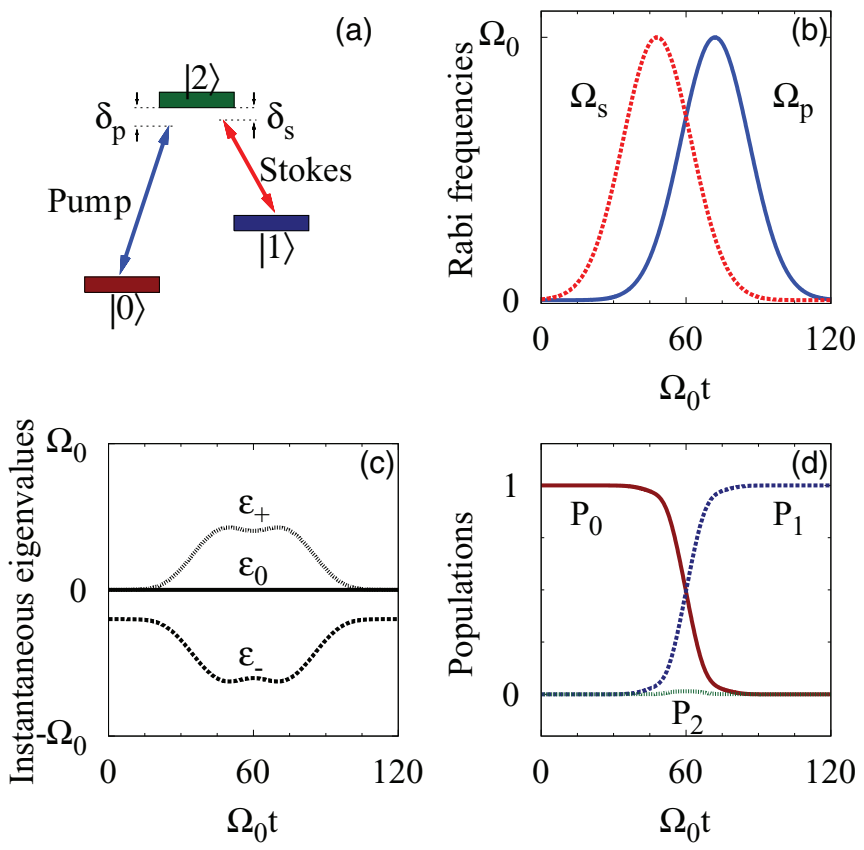

FIG. 1. (Color online) (a) Three-level system driven with ac fields in $\Lambda$ configuration. (b) The counterintuitive sequence: the Stokes field is switched on before the pump field (here $\Omega_{0} T=20, \tau=0.6 T$ ). (c) Instantaneous eigenvalues $\left\{\epsilon_{0}(t), \epsilon_{ \pm}(t)\right\}$, for $\delta=0, \delta_{p}=-0.2 \Omega_{0}$, and $\kappa=1$. (d) Population histories $\rho_{i i}(t)=|\langle i \mid \psi(t)\rangle|^{2}$ for ideal $\operatorname{STIRAP}(\delta=0)$ : the system prepared in $|0\rangle$ follows the Hamiltonian along the $\epsilon_{0}$ adiabatic path yielding complete population transfer to $|1\rangle$.

fabricable at present, provided that operating conditions and suitable design optimize the conflicting requirements of efficient coupling between states with (approximately) the same parity and protection from low-frequency noise. Second, despite of the complicated multilevel structure and of the many parameters involved, we show that the efficiency for STIRAP depends essentially on noise channels involving the trapping subspace, and determine a simple figure of merit for design and operating prescriptions of devices allowing the observation of STIRAP.

The paper is organized as follows. We introduce STIRAP in Sec. II and describe population transfer via adiabatic and nonadiabatic patterns. In Sec. III we discuss the implementation in a $\mathrm{CPB}$, and introduce the model for broadband colored noise (BBCN), extending to a $\Lambda$ system the approach introduced in Refs. 27 and 28, which quantitatively explains qubit decoherence due to BBCN in superconducting qubits. ${ }^{29-33}$ In Sec. IV we present results on the effects of the BBCN, focusing on the charge-phase regime of CPB's. In Sec. V we extend the above considerations to other regimes of the CPB and determine the figure of merit characterizing optimal design and operating conditions. In Sec. VI we compare the effects of dephasing with long memory time with Markovian dephasing, showing that in the former case driving more strongly the system would improve efficiency. Therefore STIRAP could in principle discriminate between different dynamic characteristics of decoherence sources in superconducting nanocircuits. Conclusions are drawn in Sec. VII.

\section{COHERENT POPULATION TRANSFER IN THREE-LEVEL ATOMS}

\section{A. Dark state and STIRAP}

In quantum optics STIRAP is based on a $\Lambda$ configuration [Fig. 1(a)] of two hyperfine ground states $|0\rangle$ and $|1\rangle$ and an excited state $|2\rangle$, with energies $E_{0}=0, E_{1}$, and $E_{2}$ respectively. The system is operated by two classical laser fields, ${ }^{1-4}$ the Stokes laser $\Omega_{12}=\Omega_{s} \cos \omega_{s} t$ and the pump laser $\Omega_{02}=\Omega_{p} \cos \omega_{p} t$, each being nearly resonant with the corresponding transition. The effective Hamiltonian is conveniently written in a doubly rotating frame at the angular frequencies $\omega_{k}$, where $k=p, s$ refer to pump and Stokes. In the rotating-wave approximation (RWA) the effective Hamiltonian is $^{1,4,34}$

$\tilde{H}=\delta|1\rangle\left\langle 1\left|+\delta_{p}\right| 2\right\rangle\langle 2|+\left(\frac{\Omega_{s}}{2}|2\rangle\left\langle 1\left|+\frac{\Omega_{p}}{2}\right| 2\right\rangle\langle 0|+\right.$ H.c. $)$.

Here $\delta_{s}=E_{2}-E_{1}-\omega_{s}$ and $\delta_{p}=E_{2}-E_{0}-\omega_{p}$ are the single-photon detunings, and we introduced the two-photon detuning $\delta=\delta_{p}-\delta_{s}$. Both the detunings and the Rabi frequencies $\Omega_{k}$ can be functions of time. At two-photon resonance, $\delta=0$, the Hamiltonian (1) has a zero-energy instantaneous eigenvalue $\epsilon_{0}=0$ [Fig. 1(c)] whose eigenstate is a "dark state",

$$
|D\rangle=\frac{\Omega_{s}|0\rangle-\Omega_{p}|1\rangle}{\sqrt{\left|\Omega_{s}\right|^{2}+\left|\Omega_{p}\right|^{2}}}
$$

and two other eigenstates $| \pm\rangle$ with nonzero eigenvalues $\epsilon_{ \pm}=\frac{1}{2} \delta_{p} \pm \frac{1}{2} \sqrt{\delta_{p}^{2}+\Omega_{s}^{2}+\Omega_{p}^{2}}$ whose form can be found analytically. ${ }^{1}$ If the system is in the dark state, the population is trapped in the two lowest diabatic states $\{|0\rangle,|1\rangle\}$. This is due to destructive interference of the two fields: despite excitation by the lasers the state $|2\rangle$ is never populated and no radiative decay can be detected.

By slowly varying the coupling strengths, $\Omega_{s}(t)$ and $\Omega_{p}(t)$, the dark state can be rotated adiabatically in the subspace spanned by $|1\rangle$ and $|0\rangle$. In particular STIRAP yields complete coherent population transfer $|0\rangle \rightarrow|1\rangle$ as follows: ${ }^{1}$ the system is prepared in $|0\rangle$, which coincides with the dark state for $\Omega_{s}=$ $\Omega_{p}=0$; then $\Omega_{s}$ is slowly switched on; after a delay $\tau$ also $\Omega_{p}$ is slowly switched on; at this stage $\Omega_{s}$ is slowly switched off and the dark state now coincides with $|1\rangle$; finally the protocol ends by switching off $\Omega_{p}$, achieving complete population transfer [Fig. 1(d)]. Notice that in STIRAP population transfer is achieved by a "counterintuitive" pulse sequence [Fig. 1(b)], which has several advantages. ${ }^{1,34}$ First the excited state $|2\rangle$, which may undergo strong spontaneous decay deteriorating the transfer efficiency, is never populated during STIRAP. Moreover, provided adiabaticity is preserved, STIRAP is insensitive to many details of the protocol, such as the precise timing of the operations, a property which makes it attractive for implementing fault-tolerant quantum gates. ${ }^{10,35}$

\section{B. Sensitivity to parameters}

Adiabaticity is important to achieve high efficiency since nonadiabatic effects trigger unwanted transitions detrapping the system from the dark state. A necessary condition for 


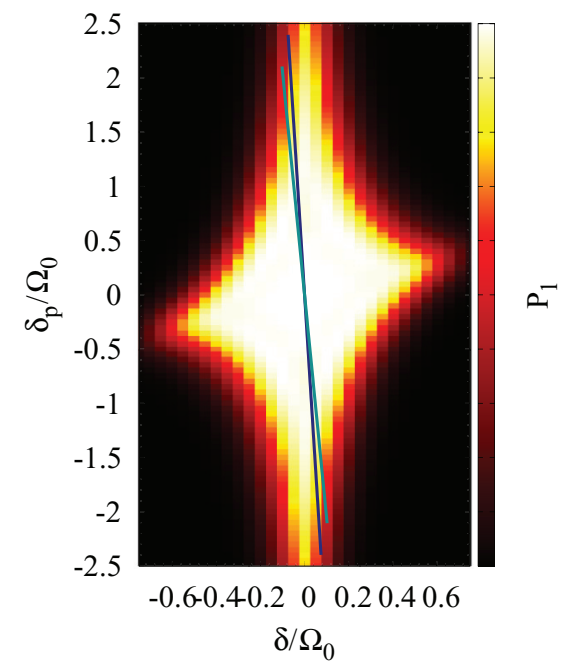

FIG. 2. (Color online) Sensitivity to detunings of the efficiency of STIRAP. Here $\Omega_{0} T=20, \tau=T / 2$, and $\kappa=1$. In the white zone the efficiency is larger than $90 \%$. Efficiency is very sensitive to a nonzero two-photon detuning $\delta$, and much less sensitive to $\delta_{p} \neq 0$ (notice the different scale of the axis). The two lines on the plot represent correlated stray detunings induced in the CPB by charge noise for two different values of $q_{g}=0.47,0.49$ (see Secs. III D and IV A).

adiabaticity is ${ }^{1} \quad\left|\left(\dot{\Omega}_{p} \Omega_{s}-\Omega_{p} \dot{\Omega}_{s}\right) /\left(\Omega_{p}^{2}+\Omega_{s}^{2}\right)\right| \ll\left|\epsilon_{ \pm}-\epsilon_{0}\right|$ which suggests that large enough Rabi peak angular frequencies $\Omega_{k}$ are needed, in order to determine a large AT splitting of the instantaneous eigenstates. We let $\Omega_{p}(t)=$ $\Omega_{0} f[(t-\tau) / T]$ and $\Omega_{s}(t)=\kappa \Omega_{0} f[(t+\tau) / T]$. A positive delay $\tau$ implements the counterintuitive sequence. For Gaussian pulses, $f(x)=\mathrm{e}^{-x^{2}}$, the choice $\tau>(\sqrt{2}-1) T$ and $\Omega_{0} T \gg 10$ yields efficient population transfer. ${ }^{34}$ As we discuss later, in superconducting nanocircuits the pump peak Rabi angular frequency $\Omega_{0}$ cannot be very large, and $T$ is limited by decoherence. We found a good tradeoff for $\Omega_{0} T=15$ and a delay $\tau=0.5 T$, which turns out to be a satisfactory choice when fluctuations of parameters are considered, and which we use unless otherwise specified.

Nonzero detunings $\delta_{s}$ and $\delta_{p}$ modify the whole adiabatic picture of STIRAP and may strongly affect the transfer efficiency. The crucial parameter is the two-photon detuning since for $\delta \neq 0$ the dark state (2) is no longer an instantaneous eigenstate and there is no adiabatic connection from the initial to the target state. As a consequence the efficiency is very sensitive to fluctuations of the two-photon detuning $\delta$, whereas large single-photon detunings $\delta_{p}$ are tolerable (see Fig. 2 and the discussion in Ref. 34). For $\delta \neq 0$ the simple picture of adiabatic passage is not valid anymore and qualitatively new phenomena occur enriching the physical scenario. In particular nonideal STIRAP may still take place via nonadiabatic transitions between adiabatic states. For small values of $\delta$, narrow avoided crossings between the instantaneous eigenvalues occur and the population is transferred by Landau-Zener (LZ) tunneling ${ }^{34}$ (see Fig. 3). For increasing $\delta$ the transfer efficiency is reduced and in general the excited state $|2\rangle$ is populated during the protocol.

It is worth stressing the importance of correlations between detunings. Indeed it is well known in atomic physics ${ }^{36}$ that if
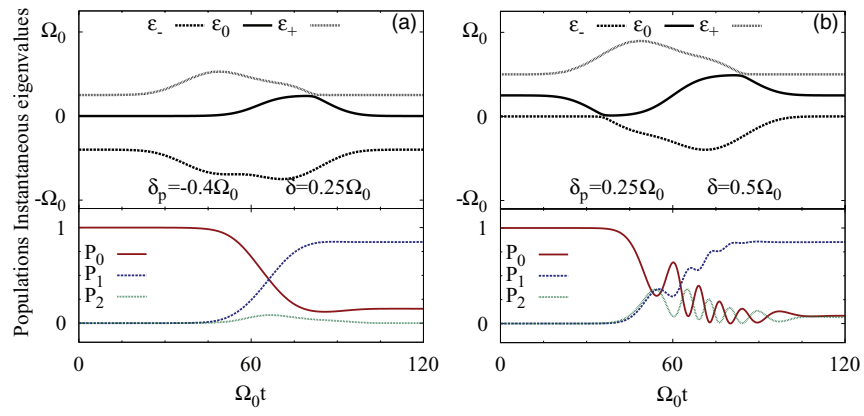

FIG. 3. (Color online) Nonideal STIRAP $\left(\Omega_{0} T=20, \tau=0.6 T\right)$ with $\delta \neq 0$, shows different classes of patterns of instantaneous eigenstates. (a) Top: instantaneous eigenstates for $\delta=0.25 \Omega_{0}$ and $\delta_{p}=-0.4 \Omega_{0}$. These LZ patterns with a single avoided crossing during the pump-induced EIT phase result from the effect of lowfrequency charge noise in CPBs (or flux noise in flux qubits). Bottom: population histories of the diabatic states. (b) Top: generic LZ pattern ( $\left.\delta=0.5 \Omega_{0}, \delta_{p}=0.5 \Omega_{0}\right)$; bottom: population histories.

$\delta_{s}$ and $\delta_{p}$ are correlated so as to nearly preserve two-photon resonance, still a large transfer efficiency is obtained. In superconducting nanodevices correlations of other nature may arise between effective fluctuations of $\delta$ and $\delta_{p}$, induced by solid-state noise. These correlations are represented by the lines in Fig. 2, and determine the typical pattern for population transfer via LZ processes ${ }^{37}$ of Fig. 3(a). Notice that in this case relatively large single-photon detunings $\delta_{p} \sim 25 \delta$ still allow coherent population transfer. This has important consequences in coherent nanodevices where fluctuations may produce large detunings $\delta_{p}$.

\section{STIRAP IN THE COOPER PAIR BOX}

\section{A. Implementation of the $\Lambda$ system}

The $\mathrm{CPB}^{21}$ is a superconducting loop interrupted by two adjacent small Josephson junctions (energy $E_{J} / 2$ ) defining a superconducting island (Fig. 4). The total capacitance $C$ gives the charging energy $E_{C}=(2 e)^{2} / 2 C$. The electrostatic energy is modulated by a gate voltage $V_{g}$, connected to the island via

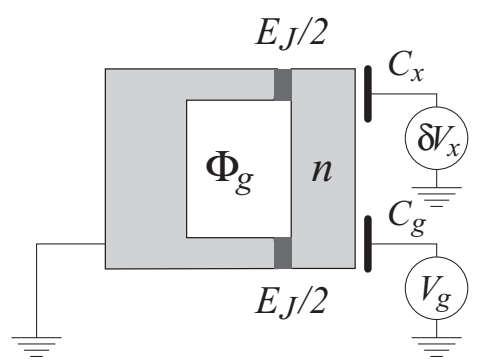

FIG. 4. In the CPB design the state of the superconducting island is a superpositions of states with a well defined number $n$ of extra Cooper pairs. The device is biased by the gate voltage $V_{g}$ determining the operating point of $q_{g}=V_{g} / 2 e C_{g}$; control is operated by an ac component of $V_{g}$. Charge fluctuations are equivalent to voltage fluctuations $\delta V_{x}$. The effective Josephson energy can be tuned via the flux $\Phi_{g}$ of the magnetic field threading the loop, $E_{J}=E_{J}\left(\Phi_{g}\right)$. 

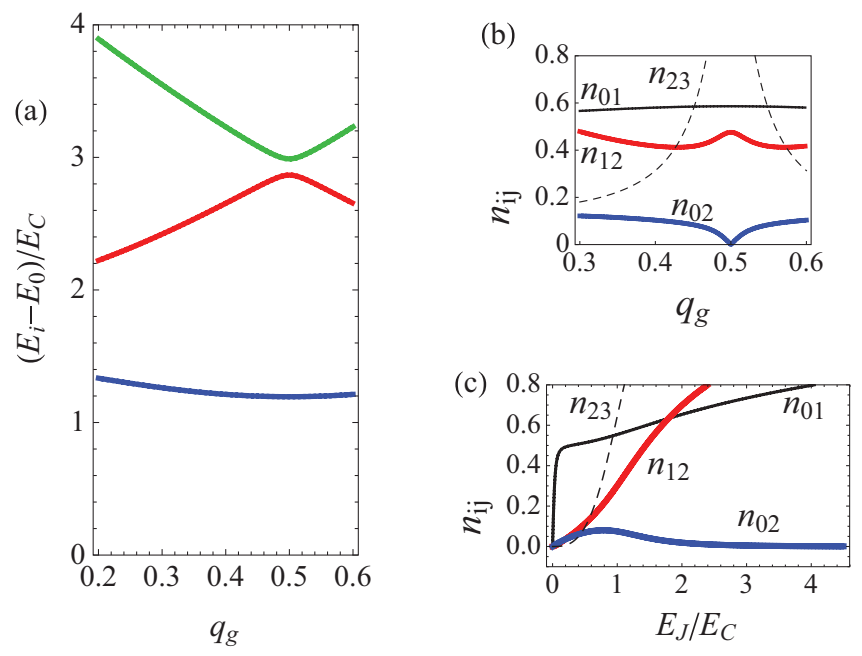

FIG. 5. (Color online) (a) Energy spectrum $E_{i}$ of a charge-phase CPB for $J=1.32$ [corresponds to the quantronium (Ref. 23)], relative to the ground state $E_{0}=0$, vs the bias $q_{g}$. (b) Matrix elements of $\hat{n}$ involved in the $\Lambda$ scheme vs $q_{g}$ for $J=1.32$ : the element $n_{02}$ vanishes at the symmetry point $q_{g}=1 / 2$; large $n_{23}$ may be a potential source of leakage from the three-level subspace. (c) Matrix elements vs $J=E_{J} / E_{C}$ for $q_{g}=0.48$; notice that $n_{02}$ is much smaller than other elements (it vanishes at $q_{g}=1 / 2$ ) and it has nonmonotonous behavior for increasing $J$.

a capacitance $C_{g} \ll C$. The Hamiltonian reads

$$
H_{0}\left(q_{g}\right)=\sum_{n} E_{C}\left(n-q_{g}\right)^{2}|n\rangle\langle n|-\frac{E_{J}}{2}(|n\rangle\langle n+1|+\text { H.c. }),
$$

where $\{|n\rangle, n \in]-\infty, \infty[\}$ are eigenstates of the number operator $\hat{n}$ of extra Cooper pairs in the island. We have defined the reduced gate charge $q_{g}=C_{g} V_{g} /(2 e)$ polarizing the island. The spectrum can be modified by choosing a specific bias $q_{g}$ (Fig. 5).

The parametric dependence of $H_{0}$ on $q_{g}$ defines a port allowing for external control of the system: by adding an ac microwave component $q_{g} \rightarrow q_{g}+q_{c}(t)$, shaped in suitable pulses, arbitrary rotations of the quantum state have been demonstrated. ${ }^{38}$

In the basis of the eigenvectors $\left\{\left|\phi_{i}\left(q_{g}\right)\right\rangle, i=0,1,2\right\}$ of $H_{0}\left(q_{g}\right)$ the driven Hamiltonian reads

$$
H(t)=\sum_{i} E_{i}\left|\phi_{i}\right\rangle\left\langle\phi_{i}\left|+A(t) \sum_{i j} n_{i j}\right| \phi_{i}\right\rangle\left\langle\phi_{j}\right|,
$$

where $n_{i j}=\left\langle\phi_{i}|\hat{n}| \phi_{j}\right\rangle$ and the control field is $A(t)=$ $-2 E_{C} q_{c}(t)$. For STIRAP we let $A(t)=\mathcal{A}_{s}(t) \cos \omega_{s} t+$ $\mathcal{A}_{p}(t) \cos \omega_{p} t$. We then transform the Hamiltonian to the doubly rotating frame, and retain only slowly varying terms, which yields the RWA (see Appendix A 1). By projecting onto the three lowest levels, $i, j=0,1,2$, we finally obtain an effective Hamiltonian $\tilde{H}$ implementing the $\Lambda$ configuration of Eq. (1), with the definitions

$$
\Omega_{p}=n_{02} \mathcal{A}_{p}, \quad \Omega_{s}=n_{12} \mathcal{A}_{s} .
$$

Therefore, $\hat{n}$ enters the peak Rabi angular frequencies, as the electric dipole does in atoms. The CPB is a tunable atom since the parametric dependence on $q_{g}$ [see Eq. (3)] affects "diabatic states", eigenenergies, and matrix elements of $\hat{n}$ (see Fig. 5). Therefore, detunings and peak Rabi frequencies in $\tilde{H}$ Eq. (1) depend on $q_{g}$.

Several superconducting qubits are based on the CPB. From the point of view of the model they differ for the values of the parameter $J=E_{J} / E_{C}$. Computational states, which are eigenstates of $H_{0}$, are superpositions of a number of "charge states" $|n\rangle$, increasing with $J$, and therefore these devices have very different energy spectra. Coherent dynamics has been observed in the charge regime ${ }^{22,25} J \ll 1$, in the charge-phase regime ${ }^{23,24} J \sim 1$, and in the phase regime ${ }^{26}$ $J \gg 1$ (from several tens up to several hundreds). Physically these devices greatly differ both in the design (size, on-chip readout scheme) and in characteristics (ease of coupling to control fields, resilience to noise), these features being crucial for functionality. Therefore the CPB allows for a thorough discussion of requirements to observe STIRAP in a wide class of nanodevices.

Notice finally that, besides transitions to higher energy levels, the external field coupling to artificial atoms may in principle trigger the $0 \rightarrow 1$ transition and for $q_{g} \neq 1 / 2$ also provides a time-dependent diagonal contribution to the effective Hamiltonian. We have shown in previous works, 9,39 where the Hilbert space of the device was exploited up to ten charge states with the full structure of the coupling to the drive, that there is no leakage in CPBs in the charge-phase regime, even in the presence of markovian noise. This is true despite of the fact that matrix element $n_{23}$ in the CPB may be large (see Fig. 5), as long as the STIRAP protocol is successfully carried without populating $|2\rangle$. Leakage from the three-level subspace is expected for $J \gg 1$, when the spectrum approaches the harmonic form; a regime where, however, STIRAP does not occur even in the three-level approximation, as we show later.

We have shown in previous works that this has no effect for $\mathrm{CPBs}$ in the charge-phase regime, due to the large anharmonicity of the spectrum, even in the presence of Markovian noise. Therefore we will safely study the effect of noise in the lowest three-level subspace. Leakage from this subspace is expected for $J \gg 1$, but in this regime STIRAP does not occur even in the three-level approximation, as we show later.

\section{B. Symmetries, decoherence, selection rules}

Tunability with $q_{g}$ has been exploited to find optimal points where qubit operations are well protected from low-frequency noise. ${ }^{23,27}$ For instance for $q_{g}=1 / 2$ the Hamiltonian (3) is symmetric for charge-parity transformations (Appendix A 2). Due to this fact CPB-based qubits biased at symmetry are well protected against external noise. This has allowed us to obtain experimental dephasing times of several hundreds of nanoseconds in charge-phase devices ${ }^{23,30}$ and ranging from $T_{2}^{*}>2 \mu \mathrm{s} J \sim 50$ in the phase regime ${ }^{26}$ up to $T_{2}^{*} \sim 0.1 \mathrm{~ms}$ recently reported. ${ }^{40}$ At the same time symmetry enforces a selection rule preventing transitions between states with the same charge-parity. In particular $n_{02}$ vanishes at $q_{g}=1 / 2$ [see Fig. 5(b)], therefore it is not possible to implement the $\Lambda$ configuration of Eq. (1), since $\Omega_{p}=0$. In Refs. 8,9 it has been proposed to overcome this problem by working slightly 
off-symmetry [see Figs. 5(b) and 5(c)], and it has been shown that the full multilevel structure of a CPB with $E_{J}=E_{C}$ allows for coherent population transfer for $q_{g} \approx 0.47$, in the presence of Markovian noise.

We stress that protection from noise and selection rules are related since they both stem from charge-parity symmetry. Notice that increasing $J$ enforces the (approximate) selection rule in a larger and larger neighborhood of the symmetry point $q_{g}=$ $1 / 2$, since it makes less effective symmetry breaking terms (asymmetric charging energy). For instance Fig. 5(c) shows that $n_{02}$ at off-symmetry $\left(q_{g}=0.48\right)$ eventually decreases for increasing $J$, making impossible the implementation of the $\Lambda$ scheme.

\section{Model for charge noise}

In principle each port of the device also allows injection of noise and provides channels for decoherence. The control port associated to $q_{g}$ couples to charge noise, and in this paper we focus on it, since it is the main source of low-frequency noise in the CPB for the regimes in which STIRAP could be observed. The structure of the coupling to noise can be obtained by allowing for fluctuations of the gate charge in the Hamiltonian Eq. (3). Their physical origin, besides voltage fluctuations of the circuit have been recognized as the effect of switching impurities ${ }^{29,41,42}$ located in the oxides or in the substrate close to the device. We let $q_{g} \rightarrow q_{g}+x$, where $x$ describes stray electrical polarization of the island, and write the resulting Hamiltonian as $H=H_{0}\left(q_{g}\right)+H_{R W}(t)+\delta H$. Here $H_{R W}(t)$ is the control Hamiltonian in the RWA, Eq. (A3), whereas $\delta H=$ $-2 E_{C} x \hat{n}$ describes fluctuations. The structure of coupling to a quantum environment is obtained on a phenomenological level by "quantizing" noise. This is obtained by letting $\delta H=\hat{X} \hat{n}+$ $H_{R}$, where $\hat{X}$ is an environment operator and $H_{R}$ describes the environment alone, and suitable counterterms. ${ }^{43}$ Markovian noise can then be studied by deriving a weak coupling quantum optical master equation (ME). However, noise in the solid state has large low-frequency components invalidating the ME. A multistage approach has been proposed ${ }^{27}$ where high- and lowfrequency noise are separated, the latter being approximated by a classical random field. Formally $\hat{X} \rightarrow \hat{X}_{f}-2 E_{C} x(t)$ where $\hat{X}_{f}$ describes fast environmental quantum degrees of freedom and $x(t)$ is a slow classical stochastic process. If we let $q_{x}(t)=q_{g}+x(t)$ the Hamiltonian is written as

$$
H=H_{0}\left[q_{g}+x(t)\right]+H_{R W}(t)+\hat{X} \hat{n}+H_{e n v} .
$$

In many cases low-frequency noise has a $1 / f$ spectrum and the leading contribution of the slow dynamics of $x(t)$ is captured by a static-path approximation (SPA), i.e., approximating the stochastic process by a suitably distributed random variable ${ }^{27,30} x$. In this simpler scenario one should first calculate the reduced density matrix $\hat{\rho}(t \mid x)$ for a given stray bias $x$ obtained by tracing out high-frequency (quantum) noise, and then averaging over the distribution $p(x)$. In particular, population histories are given by $P_{i}(t)=\int d x p(x) \rho_{i i}(t \mid x)$. Notice that for each realization $x$ of the random variable the system is prepared and measured in the eigenbasis of $H_{0}\left(q_{g}+x\right)$, which is then conveniently used to represent $\rho_{i i}$.

In the case of many weakly coupled noise sources, $p(x)$ is a Gaussian with standard deviation $\sigma_{x}$. The low-frequency noise affects the dynamics via fluctuations of energy it induces. This point of view provides a simple argument explaining why the symmetry point $q_{g}=1 / 2$ is well protected. Indeed, since at this working point the energy splitting $E_{1}$ depends only quadratically on the fluctuations $x$, energy fluctuations are suppressed. Therefore superpositions of the two lowest energy levels keep coherence for a longer dephasing time, with only a power-law suppression of the signal. ${ }^{27,30}$ This case is referred as the "quadratic noise" regime, to make a distinction with "linear noise" conditions, occurring for off-symmetry bias, where energy fluctuations are linear in $x$ yielding much stronger decoherence (Gaussian decay law).

This approach has quantitatively explained the power-law decoherence observed not only in $\mathrm{CPBs}^{30}$ but also in flux qubits, ${ }^{31}$ and has allowed finding an optimal operating point in ultrafast driven phase qubits. ${ }^{32}$ Recently it has been used to discuss properties of multiqubit systems. ${ }^{44}$ The present extension to a $\Lambda$ system of the approach of Ref. 27 enlightens us regarding the role of correlations between detunings, and provides a tool for optimal device design.

\section{Effective model for low-frequency noise in $\boldsymbol{\Lambda}$ configuration}

In order to study STIRAP the Hamiltonian (6) is projected onto the subspace spanned by the three lowest energy adiabatic eigenvectors of $H_{0}\left[q_{g}+x(t)\right]$. In doing so we assume the adiabaticity of the dynamics induced by $x(t)$, which allows us to neglect effects of the time dependence of the eigenvectors. Of course in the SPA adiabaticity of noise is automatically verified. The system plus drive Hamiltonian $H_{0}\left[q_{g}+x(t)\right]+$ $H_{R W}(t)$ in the rotated frame has the same structure of Eq. (1), but depends on the realization of the stochastic process. Fluctuations of the eigenenergies translate into fluctuations of the detunings (we let $E_{0}=0$ ). In the SPA we have

$$
\delta(x)=E_{1}\left(q_{g}+x\right)-\omega_{p}+\omega_{s} ; \delta_{p}(x)=E_{2}\left(q_{g}+x\right)-\omega_{p} .
$$

It is worth stressing that also the effective drive fluctuates, via the charge matrix element, for instance $\Omega_{p}=n_{02}\left[q_{g}+\right.$ $x(t)] \mathcal{A}_{p}$. Thus the effect of low-frequency noise in solidstate devices is conveniently recast in terms of sensitivity of the protocol to fictitious imperfections (in both phase and amplitude) of the drive. This allows ua to apply to solid state devices several results from the quantum optics realm. For instance, the known critical sensitivity to two-photon detuning translates to the fact that the main figures to be minimized, in order to achieve efficient population transfer in nanodevices, are fluctuations of the lowest energy splitting. This is a quantity which is well characterized from the qubit dynamics. ${ }^{30-33}$

\section{EFFECT OF BROADBAND COLORED NOISE ON STIRAP IN A CHARGE-PHASE CPB}

We now apply the above approach to analyze STIRAP in a CPB in the charge-phase regime $E_{J} \sim E_{C}$. An important point is that, while dephasing is minimized by operating at the symmetry point $q_{g}=1 / 2$, the selection rule $n_{02}=0$ apparently prevents implementing STIRAP. Therefore it has 
been proposed to operate slightly off symmetry, ${ }^{7,9}$ where on the other hand decoherence due to low-frequency noise increases. ${ }^{30}$ This opens the question of the tradeoff between efficient coupling of the driving fields and dephasing due to slow excitations in the solid state.

Since it is convenient to work with the largest possible pump Rabi peak frequency $\Omega_{0}$, we will consider its value as a scale. For a given peak value of $\mathcal{A}_{p}(t)$ it can be estimated as $\Omega_{0}=\Omega_{R} n_{02}\left(q_{g}\right) / n_{01}(1 / 2)$, where $\Omega_{R}$ is the maximal angular frequency for Rabi oscillations between the lowest doublet at the symmetry point, which is well characterized in experiments. We will use frequencies corresponding to $v_{R}=600 \mathrm{MHz}$, which are in principle achievable ${ }^{45}$ even if there may be technical problems in specific devices. For the Quantronium at $q_{g}=0.48$ this would correspond to a maximum $v_{p}=55 \mathrm{MHz}$.

Close to the symmetry point, coupling of the field with the Stokes transition is larger. Therefore, we could easily choose $\kappa=v_{s} / v_{p} \approx n_{12} / n_{02} \gg 1$. However, using larger values of $v_{s}$ does not improve the transfer efficiency in CPB's, ${ }^{34,46}$ therefore we will let $\kappa=1$ hereafter.

\section{A. Effects of low-frequency noise}

Low-frequency fluctuations $x$ of the gate charge determine nonexponential dephasing in qubits. ${ }^{27}$ They have been well characterized in the quantronium by Ramsey interferometry at different bias points $q_{g} \in[0.4,0.5] .{ }^{30}$ Gate charge fluctuations $\sigma_{x}$ are obtained by the measured charging energy fluctuations $\sigma_{E}=2 E_{C} \sigma_{x} \sim 0.01 E_{1}(1 / 2)$, a figure which is independent of the bias, corresponding to $\sigma_{x}=\sigma_{E} /\left(2 E_{C}\right) \approx 6 \times 10^{-3}$. These quantities are related to the integrated spectral density of the environment, ${ }^{27}$ and for $1 / f^{\alpha}$ noise they also depend on details of the protocol as the total measurement time. Even if this dependence is only logarithmic, one can take advantage of the fact that measuring the final population in STIRAP requires a lower statistics than Ramsey fringes. Therefore, for our purposes lower values of $\sigma_{x}$ are very reasonable, and hereafter we use $\sigma_{x}=0.004$. This is a realistic figure not only for the quantronium but for the whole class of CPB-based devices, since charge noise is ultimately determined by material issues which are constantly under investigation.

We consider STIRAP for the optimal conditions of nominal single and two-photon resonance, $\delta=\delta_{p}=0$. According to Eq. (7) fluctuations $x$ determine a distribution of stray detunings. For small $\sigma_{x}$ we can approximate

$$
\delta(x) \approx A_{1} x+\frac{1}{2} B_{1} x^{2},
$$

where $A_{1}=\left(\partial E_{1} / \partial q_{g}\right)=E_{C} a_{1}\left(q_{g}, J\right) \quad$ and $\quad B_{1}=$ $\left(\partial^{2} E_{1} / \partial q_{g}^{2}\right)=E_{C} b_{1}\left(q_{g}, J\right)$. It is worth stressing that arbitrary small fluctuations determine $\delta(x) \neq 0$, therefore STIRAP may occur only via nonadiabatic patterns. In the same way also $\delta_{p}(x)$ depends on the derivatives $\left(\partial^{n} E_{2} / \partial q_{g}^{n}\right)$.

In Fig. 6 we plot the populations histories $P_{i}(t)$ averaged over the fluctuations of $x$. These induce correlated fluctuations of both detunings and couplings $n_{i j}$. Device and bias parameters correspond to a quantronium biased slightly off symmetry. It is shown that low-energy fluctuations determine a $\sim 20 \%$ efficiency loss despite of the fact that protection from noise is greatly reduced. This is an interesting figure

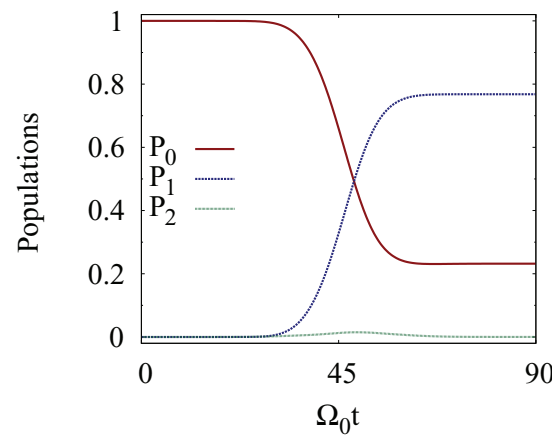

FIG. 6. (Color online) Population histories in the quantronium at $q_{g}=0.475$, averaged over fluctuations with $\sigma_{x}=0.004$. Charge fluctuations determine anticorrelated stray detunings, $\delta_{p}=-23.5 \delta$. Drives are symmetrized, $\kappa=1$. The total time for the quantronium corresponds to $\sim 250 \mathrm{~ns}$. The resulting efficiency is $P_{1}\left(t_{f}\right)=0.77$.

for superconducting nanodevices if we compare with the observed coherent population trapping of $\sim 60 \%$ recently measured in phase-type devices. ${ }^{19}$ Moreover the population of the intermediate level is very small during the whole procedure, fulfilling the requirements for coherent population transfer.

Such numerical evaluations are performed by using a fourth-order integration Runge-Kutta method for the solution of the ordinary differential equations. Convergence was tested down to a relative error lower than $10^{-3}$, by adjusting both the integration step and the number of event series. Integration over fluctuations was performed by a Monte Carlo approach with up to 5000 samples in order to attain a relative error smaller than $10^{-3}$.

Notice that the detunings depend on a single random variable $x$, therefore their fluctuations are correlated. In particular, charge noise determines anticorrelated fluctuations of effective detunings in CPBs, as is clear from the spectrum [Fig. 5(a)]. This implies that nonideal STIRAP may occur only via the typical LZ patterns ${ }^{37}$ shown in Fig. 3(a).

Notice that in the regime of Fig. 6 fluctuations of the couplings $n_{i j}$ could have been neglected. Indeed, they can be estimated from Fig. 5(b). For instance, for $J \sim 1$ and $q_{g}<0.49$, fluctuations of the amplitude of the pump pulse are $\sigma_{p} \sim a_{02} \sigma_{x} \Omega_{0}$, where $a_{02}=\partial n_{02} / \partial q_{g}$, therefore $\sigma_{p} \ll \Omega_{0}$. Numerical results (Figs. 8 and 10) actually confirm that fluctuations $n_{i j}$ yield at most corrections, and moreover when they are appreciable STIRAP does not work due to the combined effect of high-frequency noise (see Sec. IV C).

The above observation implies that for practical purposes efficiency can be discussed entirely in terms of the sensitivity to detunings. ${ }^{46}$ Diagrams in the $\left(\delta, \delta_{p}\right)$ plane (Fig. 2) can be used to understand the effect of low-frequency noise. Correlated stray detunings in the CPB are there represented by lines in the $\delta-\delta_{p}$ plane, which are straight lines for linear noise. We draw the segment corresponding to fluctuations $x \in\left[-\sigma_{x}, \sigma_{x}\right]$ (here $\left.\sigma_{x}=0.004\right)$, for each bias point $\left(q_{g}=0.47,0.49\right.$ are shown, the slope increasing by approaching $q_{g}=1 / 2$ ). If segments lie inside the light zone the efficiency is large. It is seen that efficient STIRAP requires small fluctuations $|\delta|<0.1 \Omega_{0}$ but the large anticorrelated $\left|\delta_{p}\right| \leqslant 2.5 \Omega_{0}$ is tolerable. 


\section{B. Effect of high-frequency noise}

High-frequency noise is studied by solving the quantumoptical ME in the rotating frame ${ }^{47} \dot{\rho}=\frac{i}{\hbar}[\rho, \tilde{H}]-\mathrm{D} \rho$, where $\rho$ is the density matrix and $\tilde{H}$ is the Hamiltonian (1). The structure of the dissipator $\mathrm{D} \rho$ in the basis of the diabatic states $\left\{\left|\phi_{i}\right\rangle\right\}$ reads $^{9}$

$$
(\mathrm{D} \rho)_{i j}=\frac{\gamma_{i}+\gamma_{j}}{2} \rho_{i j}-\delta_{i j} \sum_{k \neq i} \rho_{k k} \gamma_{i k}+\left(1-\delta_{i j}\right) \tilde{\gamma}_{i j} \rho_{i j}
$$

The first two terms describe emission and absorption of energy and the associated secular dephasing: $\gamma_{i j}=\gamma_{j \rightarrow i}$ are transition rates between diabatic states, and $\gamma_{i}=\sum_{k \neq i} \gamma_{k i}$ are the total decay rates of states $\left|\phi_{i}\right\rangle$. At low temperature in an undriven system only rates of spontaneous emission between diabatic states are non-negligible. In ac driven systems rates describing environment-assisted absorption are also nonzero, when the corresponding field is switched on. ${ }^{48}$ Finally the dissipator may include pure dephasing rates $\tilde{\gamma}_{i j}=\tilde{\gamma}_{j i}$.

In quantum optical systems STIRAP connects two ground states, $\gamma_{01}=\gamma_{10}=0$. Therefore as long as population in $\left|\phi_{2}\right\rangle$ is small all the transition rates act on depopulated states, and it is known that they practically do not affect population transfer. Instead in superconducting nanocircuits the decay channel $\gamma_{01}$ is active. Therefore, we expect that $\gamma_{01}$ is the main source of efficiency loss due to processes involving energy exchange with the environment. This is indeed the qualitative conclusion suggested by the results in Fig. 7 .

To clarify the physical picture in Fig. 7 we study separately the impact of adding decay channels. First we consider only spontaneous decay in the first doublet. We take $\gamma_{01} / \Omega_{0}=$ 0.01 , which is a rather large value used to emphasize the effects and we study population histories (solid lines $\rho_{i i}$ ). We find $\rho_{11}(t) \approx P_{1}(t) \mathrm{e}^{-\gamma_{01}\left(t-t_{i}\right)}$, where $P_{1}(t)$ is the population in
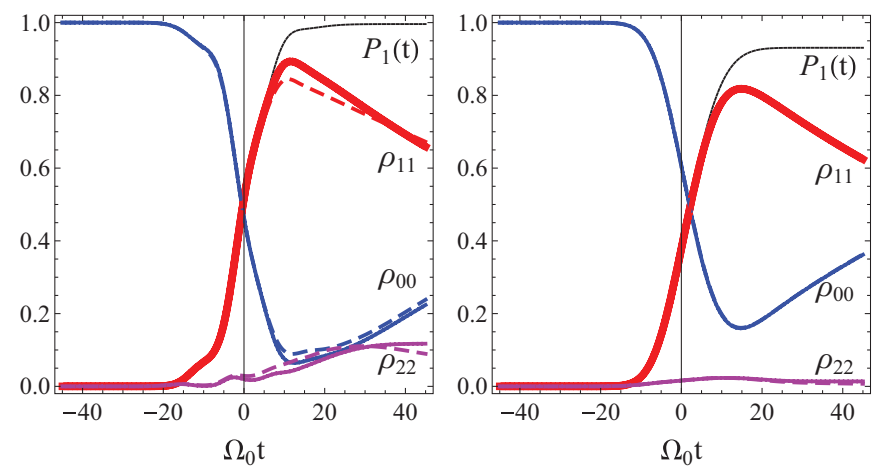

FIG. 7. (Color online) Population histories $\rho_{i i}(t)$ in the presence of high-frequency noise ( $P_{1}$ is in absence), at resonance ( $\delta=\delta_{p}=0$, left panel) and for finite anticorrelated detunings ( $\delta=0.05, \delta_{p}=$ $-25 \delta$, right panel), for $\kappa=1$. Solid lines are obtained by inserting only $\gamma_{01}=1 / T_{1}$ in Eq. (8), describing relaxation $1 \rightarrow 0$ only, and the associated secular dephasing. We have chosen a rather large $\gamma_{01} / \Omega_{0}=$ 0.01 to emphasize the effect. Dashed lines take into account all the other low-temperature emission and drive-induced absorption channels (the chosen rates overestimate these processes), which are seen to have a limited impact on the efficiency. Physical scales for $\Omega_{0}=3.46 \times 10^{8} \mathrm{rad} / \mathrm{s}$ (the value we use for the Quantronium at $\left.q_{g}=0.48\right)$ are $T \approx 43 \mathrm{~ns}$, for the overall protocol $T_{T} \approx 290 \mathrm{~ns}$, and the chosen $T_{1} \approx T_{T}$. absence of noise; therefore this channel mainly determines the simple population loss $1 \rightarrow 0$ when the target state is populated. It also determines a nonvanishing population $\rho_{22} \neq 0$ which indicates detrapping from the dark state due to loss of coherence.

Adding all the other decay channels (dashed lines) produces minor modifications of this picture (Fig. 7 left panel) for fields at resonance. No modification at all occurs for nonvanishing detunings, mimicking low-frequency fluctuations. The reason is that in this latter case $\gamma_{01}$ does not determine substantial detrapping, and population of $\left|\phi_{2}\right\rangle$.

In detail, results of Fig. 7 were obtained by using rates for the other decay channels which overestimate unwanted processes, namely $\gamma_{12}=2 \gamma_{01}$, whereas $\gamma_{02}=0.2 \gamma_{01}$ (accounting for the suppression by selection rules). Notice that we do not take into account the fact that these emission rates become smaller when the drive amplitudes $\Omega_{k}(t)$ are large enough, as resulting from the generalized (Bloch-Redfield) ME for ac driven systems undergoing Rabi oscillations. ${ }^{48}$ This latter approach shows that also in the weak damping Rabi regime $\left(T \Omega_{k}(t) \gg 1\right.$ and $\left.\delta_{k} \ll \Omega_{k}(t)\right)$ field-induced absorption sets in, even at low temperatures. We take into account this channel phenomenologically, letting $\gamma_{21}(t)=\gamma_{12} / 4[1-$ $\left.\delta_{s} /\left(\sqrt{\delta_{s}^{2}+\Omega_{s}^{2}(t)}\right)\right]^{2} g\left[\Omega_{s}(t) T\right]$, where $g(x) \approx 1$ only for $x \gg$ 1 accounts for the requirement that field-induced processes set in for underdamped Rabi oscillations. We used a similar expression for $\gamma_{20}(t)$.

Notice that while secular dephasing is taken into account in Fig. 7 we did not include Markovian pure dephasing rates, $\tilde{\gamma}_{i j}=0$. Indeed, we argue that pure dephasing comes mainly from low-frequency (non-Markovian) noise accounted for by classical fluctuations of $x$. In the next section we study the combined effect of high and low-frequency noise. We will discuss different models of pure dephasing in Sec. VI. We finally mention that, for charge-phase CPB, it has been shown that operating at $q_{g}=0.48$ already provides sufficient coupling $n_{02}$ to observe STIRAP in the presence of the Markovian component of noise ${ }^{39}$

\section{Combined effect of low- and high-frequency noise}

The main conclusion of the last two sections is that the leading effects reducing coherent population transfer in nanodevices essentially involve decoherence of the first doublet. Another detrimental effect is that coupling to the pump pulse may be too weak due to (approximate) parity selection rules at (near) the symmetry point.

With this in mind we investigate the interplay of low- and high-frequency fluctuations, for $q_{g} \leqslant 1 / 2$ in a charge-phase CPB. Indeed we will argue in the next section that STIRAP can be observed only in this regime. Here we consider a case-study device such as the quantronium, where noise in the first doublet has been well characterized. We take the value $T_{1}=1 \mu \mathrm{s}$ which is achievable in the class of CPB devices at $q_{g}=1 / 2$, and neglect its weak dependence on the bias. ${ }^{30}$

Results are summarized in Fig. 8 where the efficiency is plotted against the bias $q_{g}$, showing the impact of adding various low-frequency and high-frequency decoherence channels. Curves refer to the same $\Omega_{0} T=15$, which guarantees adiabaticity for ideal STIRAP. It is apparent the different 


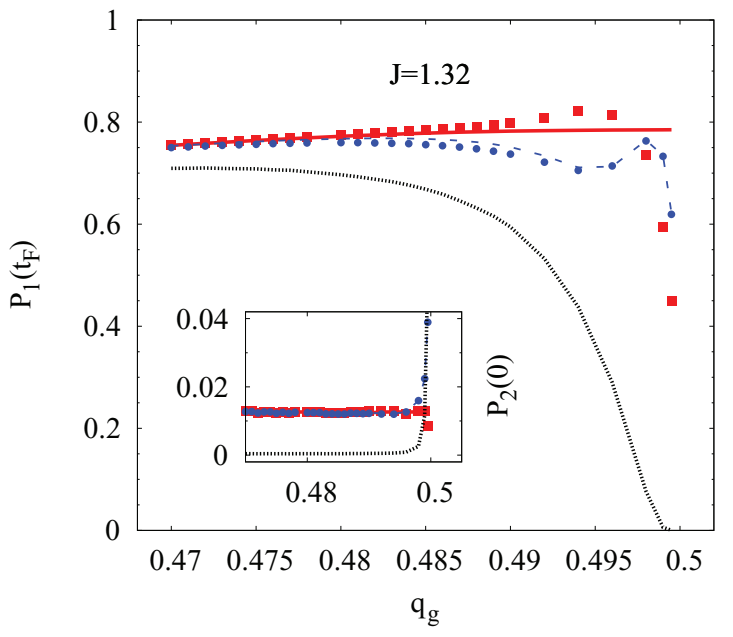

FIG. 8. (Color online) Efficiency of STIRAP $P_{1}\left(t_{f}\right)$ as a function of the bias $q_{g}$ in the presence of low-frequency and BBCN for the quantronium $\left(E_{J} / E_{C}=1.32\right)$. Here $\Omega_{0} T=15, \sigma_{x}=0.004, v_{01}=$ $600 \mathrm{MHz}$. Upper curves show effects of low-frequency noise, whereas the lower curve (black dashed) includes also high-frequency noise. Low-frequency noise is analyzed by adding different components, namely linear and quadratic correlated fluctuations of detunings (red solid curve and red squares), linear and quadratic fluctuations of $n_{02}$ (blue solid curve and blue dots). For off-symmetry bias $\left(q_{g}<\right.$ 0.9 ), only linear detuning noise is important (see Appendix B for the behavior near $q_{g}<1 / 2$ ). In the inset the population $P_{2}(0)$ at intermediate times is shown.

behavior sufficiently far $\left(q_{g}<0.49\right)$ and close $\left(q_{g} \approx 0.5\right)$ to the symmetry point.

For off-symmetry bias it is possible to observe STIRAP despite of the reduced protection from low-frequency noise. In this regime low-frequency noise is the main source of efficiency loss, allowing a population transfer close to $\sim 80 \%$. Notice that only linear fluctuations of the detunings are important; indeed Fig. 8 shows that accounting for the whole structure of low-energy fluctuations yields basically the same result, as the dependence of $E_{10}$ and $n_{02}$ on $q_{g}<0.49$ would a priori suggest. Efficiency is reduced to $\sim 70 \%$ when also effects of high-frequency noise are taken into account (black dashed curve in Fig. 8).

Instead by approaching $q_{g}=1 / 2$, while low-frequency fluctuations would still allow for some population transfer, the interplay with high-frequency noise, mainly due spontaneous decay $|\phi\rangle_{1} \rightarrow\left|\phi_{0}\right\rangle$, leads to the suppression of the efficiency (solid curve in Fig. 8). Actually, in this regime the description of the effect of low-frequency fluctuations is more complicated, spoiling the simple picture based on sensitivity to detunings. We discuss in Appendix B all of the information contained in Fig. 8. The main point is the observation that, even if the device is biased at $q_{g}=1 / 2$, fluctuations still allow for a nonvanishing pump coupling despite the parity selection rule. The reason why high-frequency noise suppresses the efficiency is understood by recalling that STIRAP requires large pulse area, $\Omega_{p}\left(q_{g}+x\right) T \gg 10$. Since close enough to the symmetry point $\Omega_{p}$ becomes small, larger and larger $T$ are needed which eventually exceed by far $T_{1}$. This mechanism explains the fact that the loss of efficiency due to high-frequency noise (Figs. 8 and 10)

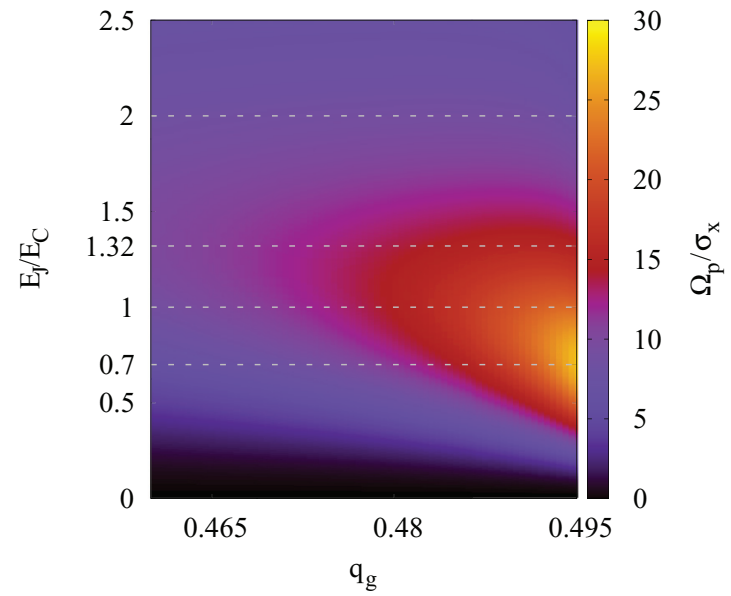

FIG. 9. (Color online) The figure of merit $\Omega_{p} / \sigma_{\delta}$ is plotted in the $\left(q_{g}, E_{J} / E_{C}\right)$ plane. We have chosen $\sigma_{x}=0.004$ and $\Omega_{0}$ produced by an external field, which would determine Rabi oscillations with $v_{R}=600 \mathrm{MHz}$ in the first doublet. The analysis is valid far enough from the charge-parity symmetry point, which is not an interesting regime since the efficiency is suppressed. Dashed lines correspond to the values of $E_{J} / E_{C}$ checked in this paper (Figs. 8 and 10).

appears to depend strongly on $q_{g}$, even if we neglected the (in any case weak) dependence of the rates $\gamma_{i j}$ on the bias.

The population $P_{2}(0)$ of the intermediate level during the adiabatic passage phase remains small in the presence of $\mathrm{BBCN}$ (inset of Fig. 8). This is an essential requirement for success and applications of the protocol, and completes the statement that STIRAP should be observable in charge-phase CPBs.

Finally we mention that working with larger asymmetry, while providing a stronger pump coupling (see Fig. 5), enhances the effect of low-frequency noise, reducing the overall efficiency. This is apparent from the trend in Fig. 8 (see also Fig. 9), indicating that optimization of strong enough pump coupling and protection from low-frequency noise is a key issue for the implementation of a $\Lambda$ system.

\section{OPTIMAL DESIGN OF THE DEVICE}

Efficiency of population transfer may be improved by optimizing the parameters of the protocol. In the last section we have shown that, due to the combined effect of the approximate symmetry and of spontaneous decay, efficiency is large enough only if the device is biased slightly away from the symmetry point. In this section, we argue that in this regime one should mainly optimize the tradeoff between coupling of the pump pulse and energy fluctuations of the lowest doublet of the device, due to low-frequency noise. Indeed the relevant figure of merit turns out to be

$$
\frac{2 E_{C}\left\langle n_{02}\right\rangle}{\sigma_{\delta}} \propto \frac{\Omega_{p}^{\max }}{\sigma_{\delta}},
$$

where $\sigma_{\delta}=\sqrt{A_{1}^{2} \sigma_{x}^{2}+\frac{1}{2} B_{1}^{2} \sigma_{x}^{4}}$ are the fluctuations of $\delta(x)$. Efficient population transfer requires large enough values of this quantity, which depends on $q_{g}$ and on $J$, via $\left\langle n_{02}\right\rangle$ and $\left(A_{1}, B_{1}\right)$. This allows us to choose convenient design and operating conditions (see Fig. 9). 
The criterion is clear from heuristic grounds, but can also be justified starting from an estimate of the linewidth for population transfer at finite $\delta$. To this end we generalize an argument given by Vitanov et al. $^{34}$ They noticed that, even if states of the adiabatic basis $\{|D\rangle,| \pm\rangle\}$ are not anymore instantaneous eigenstates, still $|D\rangle$ provides a connection between the diabatic states $\left|\phi_{0}\right\rangle$ and $\left|\phi_{1}\right\rangle$. Then it is argued that efficiency loss depends on processes triggering transitions from $|D\rangle$ to $| \pm\rangle$. These are due to nonvanishing off-diagonal entries of the Hamiltonian in the adiabatic basis, which are proportional to $\delta$. Therefore, if $\delta \gg \min \left|\epsilon_{ \pm}\right|$population transfer does not occur. This condition implies that for $\delta_{p}=0$ the linewidth scales linearly with the amplitude of the fields, ${ }^{34}$ $\delta_{\frac{1}{2}}=d(\tau) \sqrt{\left(\Omega_{p}^{\text {max }}\right)^{2}+\left(\Omega_{s}^{\text {max }}\right)^{2}}$. In our case stray detunings $\delta(x)$ and $\delta_{p}(x) \neq 0$ are anticorrelated, therefore leakage from $|D\rangle$ occurs during the pump phases [see Fig. 3(a)]. Moreover, from Fig. 2 we see that it is substantial only when $\delta_{p}>2 \Omega_{0}$. In this regime the relevant condition $\delta_{\frac{1}{2}}=\left|\epsilon_{-}\right|$is an equation whose solution can be still written as $\delta_{\frac{1}{2}} \approx d^{\prime}(\tau, \kappa) \Omega_{p}^{\max }$. Asking that fluctuations of $\delta$ do not destroy the efficiency means that we need $\sigma_{\delta} \ll \delta_{\frac{1}{2}}$. Therefore, we need large values of the parameter $\delta_{\frac{1}{2}} / \sigma_{\delta} \propto \Omega_{p}^{\max } / \sigma_{\delta}$, which justifies the figure of merit defined in Eq. (9). Our derivation does not take into account fluctuations of the matrix elements, since they are negligible in the regime where STIRAP could work. For the same reason we did not include in Fig. 9 the region near $q_{g}=1 / 2$, since in this regime STIRAP is in any case prevented by spontaneous decay, due to the too low achievable values of $\Omega_{0} T_{1}$.

We check the optimization suggested by Fig. 9 by looking at STIRAP for different values of $J$. It is seen that proper fabrication parameters allow us to obtain larger efficiency [ $J=0.7$ in Fig. 10(a) and $J=1$ in Fig. 10(b)]. Good external parameters are those used in Fig. 8, namely $\Omega_{0} T=15$ and $v_{01}=600 \mathrm{MHz}$, and $q_{g} \approx 0.48$, corresponding to a figure of merit of about 20. Instead for larger values of $J$, as in the Transmon ${ }^{26}$ design, pump coupling is insufficient even if protection against noise is much better. In the opposite limit of charge qubits $E_{J} / E_{C} \ll 1$, the efficiency is also small because of both small coupling and reduced protection from noise. This latter strongly suppresses population transfer also for a bias $q_{g}$ far off symmetry, despite the fact that coupling to the field increases. Notice that the dependence of the efficiency on the parameter $J$, besides providing prescriptions for the fabrication, can also be checked by on-chip tuning of $E_{J}$ via an external magnetic flux $\Phi_{g}$ (see Fig. 4).

\section{COMPARISON OF DIFFERENT MECHANISMS OF DEPHASING}

Studying low-frequency noise in nanodevices by a nonMarkovian model is necessary to explain quantitatively striking experimental features observed in quantum bits, such as the peculiar nonexponential initial decoherence. ${ }^{30-32}$ Moreover, this approach provides valuable additional information such as relations between effects of noise for different bias point ${ }^{30-32}$ and different device design, ${ }^{33}$ which are uniquely explained by the parametric dependence of the energy spectrum. We stress
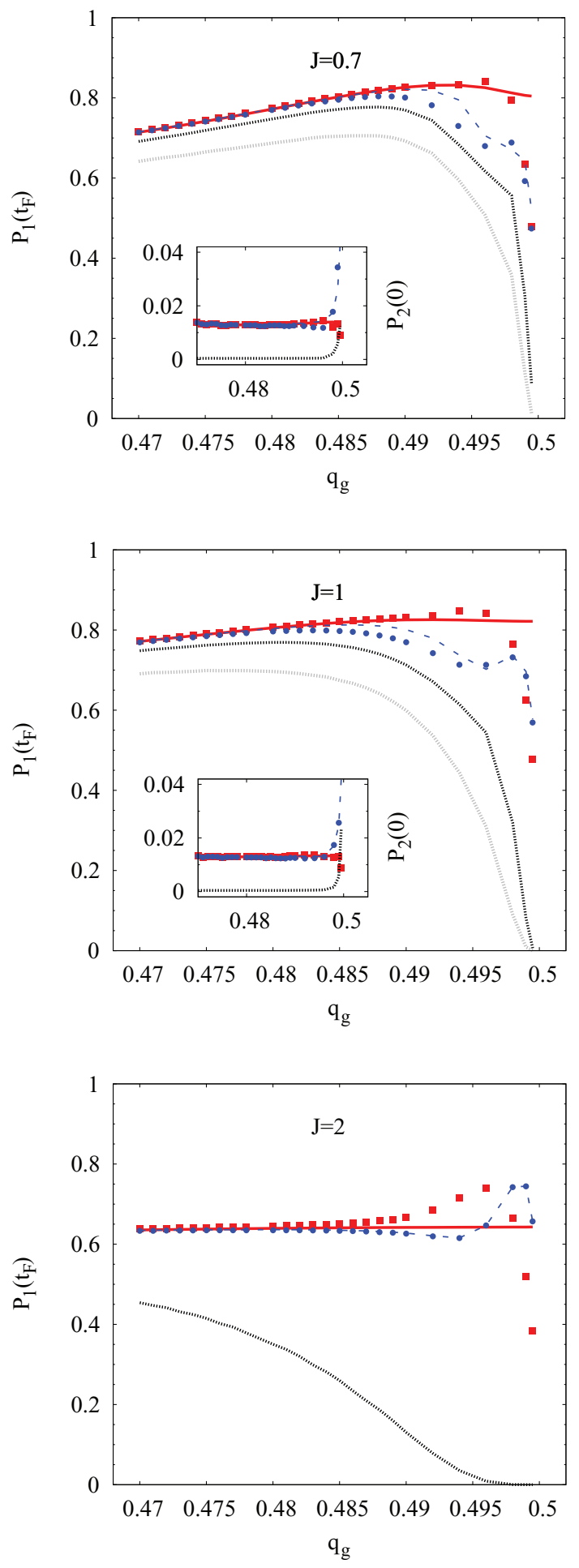

FIG. 10. (Color online) Efficiency $P_{1}\left(t_{f}\right)$ vs bias $q_{g}$ for $J=$ $0.7,1,2$ (see Fig. 9). Parameters are the same as in Fig. 8, where $T_{1}=1000 \mathrm{~ns}$ (black short-dashed curves). In the two upper panels efficiency for smaller $T_{1}=500 \mathrm{~ns}$ (gray short-dashed curve) is also shown.

that such a picture is entirely due to the non-Markovian nature of BBCN.

Therefore, this work complements previous studies in the quantum optics realm where typically the Markovian ME is used. In this latter approach pure dephasing is studied 


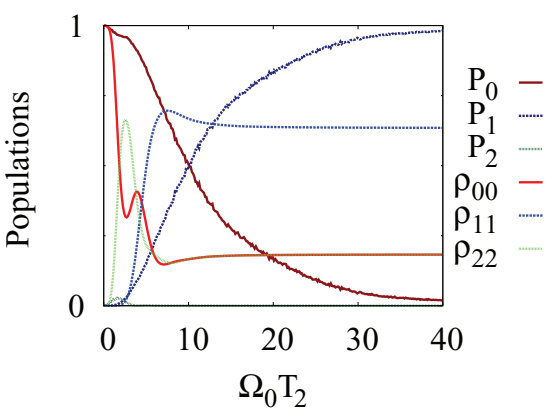

FIG. 11. (Color online) Efficiency of STIRAP (final populations) as a function of the drive amplitudes $\Omega_{0}$. We compare the case of Markovian $\left(\rho_{i i}\right)$ pure dephasing ${ }^{49}$ with the non-Markovian $\left(P_{i}\right)$ model studied here. In both cases we let $T=T_{2}=57 \mathrm{~ns}$, which for non-Markovian noise is obtained by taking $\sigma_{x}=0.004$ in a device with $J=1.32$ at $q_{g}=0.48$. It is seen that the effects of non-Markovian dephasing can be attenuated and suppressed by using larger $\Omega_{0}$, whereas for Markovian noise STIRAP, when effective, does not depend on $\Omega_{0}$.

by considering only nonvanishing dephasing rates $\tilde{\gamma}_{i j}$ in the dissipator Eq. (8), instead of the static fluctuations considered in this paper. Pure dephasing in the Markovian ME was studied by Ivanov et al. ${ }^{49}$ who derived an adiabatic solution of the Liouville equation interpolating between the coherent and the incoherent limit. They predicted striking behaviors as a function of the control parameters, deriving several analytic results, which have been numerically checked. In particular, for Gaussian pulses, populations at the end of the protocol were found to be

$$
\begin{aligned}
& \rho_{11}(\infty)=\frac{1}{3}+\frac{2}{3} e^{-3 \tilde{\gamma}_{01} T^{2} /(8 \tau)}, \\
& \rho_{00}(\infty)=\rho_{22}(\infty)=\frac{1}{3}-\frac{1}{3} e^{-3 \tilde{\gamma}_{01} T^{2} /(8 \tau)}
\end{aligned}
$$

Notice that in this approximation the efficiency is determined by the dephasing rate of the lowest doublet only, $\tilde{\gamma}_{01}$. The conclusion that other dephasing channels are less relevant (actually for $\tilde{\gamma}_{12}, \tilde{\gamma}_{02} \gg \tilde{\gamma}_{01}$ some dependence appears in the numerical solutions of the ME) agrees qualitatively with our results with the static fluctuator model. In contrast, the other striking feature of Eq. (10), namely that losses due to dephasing are independent on the peak Rabi frequencies, does not hold for low-frequency noise. Following Ref. 49 we plot in Fig. 11 populations $\rho_{i i}(\infty)$ obtained numerically from the Markovian ME, using fixed $T=T_{2}^{*}=1 / \tilde{\gamma}_{01}$, for increasing pulse amplitude $\Omega_{0}$. We compare them with the populations $P_{i}(\infty)$ for the BBCN non-Markovian model, where linear fluctuations of the detunings are considered such that $\sigma_{x}=\sqrt{2} /\left(A_{1} T_{2}^{*}\right)$, which yield the same $T_{2}^{*}$ in the qubit dynamics. It is seen that efficiency for BBCN depends on $\Omega_{0}$ and improves for increasing values.

Dependence on $\Omega_{0}$ is a natural consequence of nonideal STIRAP occurring via LZ patterns determined by lowfrequency noise. Markovian noise cannot account for this scenario. The situation here is reminiscent of dynamical decoupling $^{50}$ which eliminates dephasing for $1 / f$ noise sources, as the effect of a strong continuous ac fields also does. $^{51}$
Another difference between Markovian and non-Markovian dephasing is that this latter practically does not populate the intermediate level $\left|\phi_{2}\right\rangle$, although it decreases the transfer efficiency. This is another indication of the reduced sensitivity of the protocol to low-frequency noise. In contrast, sensitivity of $\rho_{22}$ to Markovian noise is substantial and could give direct information on $\tilde{\gamma}_{01}$, as seen from Eq. (10). This observation is reminiscent of the proposal of Ref. 5 of using EIT to probe decoherence of a phase qubit based on a superconducting quantum interference (SQUID) nanodevice. Having in mind realistic noise spectra, it is likely that the contribution of intermediate frequencies may determine effects similar to Markovian dephasing. Therefore, cross-checking measurement of decoherence of two- and three-level dynamics could give valuable spectral-resolved information on the environment.

We stress the striking implication of the non-Markovian nature of the noise, namely correlations between fluctuations of the detunings, entirely determined by the parametric dependence of the energy spectrum. Effects of time-correlated (Ornstein-Uhlembeck) phase noise in optical systems were studied by Monte Carlo simulations in Ref. 36, where the regime of partially correlated $\delta_{p}$ and $\delta_{s}$ was addressed. In nanodevices the situation is different since we have strongly anticorrelated (or correlated) stray detunings $\delta$ and $\delta_{p}$. It would be interesting to investigate dynamic phase diffusion also in this case.

\section{CONCLUSIONS}

In this paper we have studied the combined effect of lowfrequency and high-frequency charge noise on the coherence of a CPB operated as a three-level artificial atom in a Lambda configuration. Observation of STIRAP should be possible in devices within present fabrication standards, provided both design and operating conditions are carefully chosen.

We have shown that efficient population transfer requires optimizations of the tradeoff between large enough pump coupling and the implied larger sensitivity to low-frequency noise. To this end the CPB should be biased slightly off symmetry in a region where low-frequency fluctuations of the energy spectrum are linear in the fluctuations $x$ of the control parameter $q_{g}$.

We have shown that the noise is conveniently analyzed by mapping it onto fictitious correlated fluctuations of the detunings (see Fig. 2). This simple picture emerges because, despite the complications brought about by the multidimensional space of parameters, the efficiency for STIRAP is shown to depend essentially on noise channels relative to the trapped subspace only. The relevant channels can be fully characterized by operating the nanodevice as a qubit, as in Refs. 29-33.

We have found that the tradeoff is summarized by a single figure of merit, given in Eq. (9), which indicates favorable conditions for observation of STIRAP. Its remarkable dependence on features of the three-level spectrum of the device (energy correlations, symmetries) suggests that band structure engineering may play a key role in determining optimal design solutions. This analysis, together with other already available tools, such as improvements in materials and control circuits, 
and a systematic investigation of parameters and pulse crafting, guarantees room for further improvement of the efficiency.

In this work we did not consider other noise sources (such as the readout circuit or critical current noise), which are possibly coupled to the device in channels "orthogonal" to the drive. This is because in the successful regime for STIRAP they lead to minor effects in CPBs. ${ }^{30}$ They can be easily accounted for by a slight generalization of our approach, allowing for independent noise sources. Notice that each noise source could determine its own correlations of $\delta$ and $\delta_{p}$.

We remark that the physical picture emerging from this work applies to the whole class of superconducting nanocircuits used so far for implementing quantum bits. ${ }^{20}$ Our full analysis applies to flux-qubits ${ }^{52}$ where a coordinate-parity selection rule holds ${ }^{7}$ and a symmetry point exists, except that two orthogonal noise sources (flux and critical current plus charge $^{31}$ ) should be taken into account for accurate predictions. It also applies to phase-qubits ${ }^{53}$ where only linear fluctuations are important, ${ }^{54}$ but detunings are differently correlated. In all these devices the figure of merit analogous to that of Eq. (9) can be used to characterize the effect of low-frequency noise versus efficient coupling.

A natural extension of our work is the investigation of dynamic diffusion for correlated phases and/or detunings in the experimentally relevant case of $1 / f^{\alpha}$ noise, and to which extent the dependence on the drive intensity of the resilience to low-frequency noise can be used for some effective dynamical decoupling. Moreover, it has been pointed out that the presence of one or few more strongly coupled fluctuators may deteriorate the efficiency of ideal STIRAP 55 and it would be interesting to extend the investigation to the LZ scenario. Finally circuit-QED ${ }^{24}$ based architectures are natural candidates for the implementation of STIRAP with quantum fields, the physics related to $\mathrm{BBCN}^{56}$ must be studied in this broader scenario.

\section{ACKNOWLEDGMENTS}

One of us (G.F.) acknowledges E. Arimondo for having pointed out the mechanism of efficiency improvement by larger pulse amplitudes in nonideal STIRAP. We thank Franco Nori for useful comments. This work was partially supported by the EU through Grant No. PITN-GA-2009-234970, and by MIUR through Grant. No. PON02_00355_3391233, "Tecnologie per l'ENERGia e l'Efficienza energETICa ENERGETIC".

\section{APPENDIX A: MORE ON THE CPB}

\section{Driven three-level effective Hamiltonian}

Manipulation of the quantum state is performed by adding to the dc part of the gate voltage ac microwave pulses with small amplitude, $q_{g} \rightarrow q_{g}+q_{c}(t)$. The resulting Hamiltonian can be written as

$$
H(t)=H_{0}\left(q_{g}\right)+A(t) \hat{n},
$$

where $A(t)=-2 E_{C} q_{c}(t)$. The effective three-level artificial atom Hamiltonian is obtained by projecting $H(t)$ onto the subspace spanned by the three lowest energy eigenvectors $\left|\phi_{i}\right\rangle$, $i=0,1,2$, of $H_{0}\left(q_{g}\right)$,

$$
H(t)=\sum_{i} E_{i}\left|\phi_{i}\right\rangle\left\langle\phi_{i}\left|+A(t) \sum_{i j} n_{i j}\right| \phi_{i}\right\rangle\left\langle\phi_{j}\right|,
$$

where $n_{i j}=\left\langle\phi_{i}|\hat{n}| \phi_{j}\right\rangle$. The STIRAP protocol can be carried out if we let $A(t)=\mathcal{A}_{s}(t) \cos \omega_{s} t+\mathcal{A}_{p}(t) \cos \omega_{p} t$. We then perform the RWA, by retaining only quasiresonant offdiagonal and corotating terms of the drive, which simplifies to

$$
\begin{aligned}
A(t) \hat{n} \rightarrow H_{R W}(t)= & \frac{1}{2}\left[n_{12} \mathcal{A}_{s}(t) \mathrm{e}^{i \omega_{s} t}\left|\phi_{1}\right\rangle\left\langle\phi_{2}\right|\right. \\
& \left.+n_{02} \mathcal{A}_{p}(t) \mathrm{e}^{i \omega_{p} t}\left|\phi_{0}\right\rangle\left\langle\phi_{2}\right|\right]+ \text { H.c. }
\end{aligned}
$$

Finally, the Hamiltonian is transformed to the doubly rotated frame, at angular frequencies $\omega_{s}$ and $\omega_{p}$ via the transformation $U_{r f}=\exp \left[i\left(\omega_{s}\left|\phi_{1}\right\rangle\left\langle\phi_{1}\left|+\omega_{p}\right| \phi_{0}\right\rangle\left\langle\phi_{0}\right|\right) t\right]$. This yields an effective Hamiltonian $\tilde{H}\left(q_{g}\right)$ with the structure of Eq. (1), implementing the $\Lambda$ configuration. Notice that $n_{i j}=\left\langle\phi_{i}|\hat{n}| \phi_{j}\right\rangle$ play the same role of the dipole matrix elements in the definition Eq. (5) of the Rabi frequencies.

\section{Charge-parity symmetry and selection rules}

Charge parity is a possible symmetry of wave functions in charge space which emerges because of the discrete nature of the momentum. Formally we introduce operators $\Pi_{q}=\sum_{n}|q-n\rangle\langle n|$, which implement a reflection and then a translation in the charge space. If the parameter $q$ is integer $\Pi_{q}$ always operates onto the same Hilbert state of discrete charges. It is easy to see that

$$
\begin{aligned}
\Pi_{q}^{-1} & {\left[\sum_{n}\left(n-q_{g}\right)^{2}|n\rangle\langle n|\right] \Pi_{q}=\sum_{n}\left(n-q+q_{g}\right)^{2}|n\rangle\langle n|, } \\
\Pi_{q}^{-1}\left[\sum_{n}|n\rangle\langle n \pm 1|\right] \Pi_{q} & =\sum_{n}|n\rangle\langle n \mp 1| .
\end{aligned}
$$

Therefore, one can seek for the invariance of the family of Hamiltonians (3). Symmetry points are found for $q_{g}=$ $q / 2$, where $H_{0}\left(q_{g}\right)$ is invariant with respect to $\Pi_{2 q_{g}}$. Since $\Pi_{q}^{2}=\mathbb{1}$, for symmetric $H_{0}$, eigenvalues can be chosen with a well defined charge parity $\Pi_{2 q_{g}}\left|\phi_{j}\left(q_{g}\right)\right\rangle=(-1)^{j}\left|\phi_{j}\left(q_{g}\right)\right\rangle$, and parity selection rules hold such that for states of different parity charge matrix elements vanish, $\left\langle\phi_{j}|n| \phi_{i}\right\rangle=0$.

\section{APPENDIX B: FLUCTUATIONAL BEHAVIOR NEAR THE SYMMETRY POINT}

We give a more detailed account on the effects of lowenergy fluctuations close to $q_{g}=1 / 2$, displayed in Figs. 8 and 10 , and on how they combine with high-frequency noise. On approaching $q_{g}=1 / 2$, fluctuations of the detunings turn from linear to quadratic. These fluctuations alone (thick red squares in the figures) would determine a nonmonotonic behavior of the efficiency on approaching the symmetry point. Indeed for $0.49 \lesssim q_{g} \lesssim 0.495$ fluctuations for $x>0$ yield smaller stray detuning $\delta\left(q_{g}+x\right)$ than in the linear approximation, and the efficiency increases. However, approaching the symmetry point fluctuations $\langle|\delta|\rangle$ exceed the linewidth $\delta_{\frac{1}{2}}$. Indeed, since this latter scales with $\Omega_{0}$ (see Sec. V) and thus vanishes for $q_{g} \rightarrow 1 / 2$, we find $\langle|\delta|\rangle / \Omega_{0} \rightarrow \infty$ and the efficiency 
should eventually vanish. However, in this regime also the effect of fluctuations of the couplings play a role. We study these fluctuations in linear and quadratic approximation, $n_{i j}\left(q_{g}+x\right) \approx n_{02}\left(q_{g}\right)+A_{02} x+\frac{1}{2} B_{02} x^{2}$. It turns out that only fluctuations of $n_{02}$ are possibly relevant, and only in the regime where fluctuations of $\delta$ 's are quadratic. However, in this regime they spoil the picture based on fluctuations of $\delta$ 's only. Indeed, for $0.49 \lesssim q_{g} \lesssim 0.495$ smaller values of $\Omega_{p}\left(q_{g}+x\right)$ for $x>0$ compensate the positive effect of smaller $\delta\left(q_{g}+x\right)$. In contrast, on approaching the symmetry point slow fluctuations of $n_{02}$ provide a nonvanishing coupling which is enough to yield a nonzero efficiency. Notice that this is true even at the nominal bias $q_{g}=1 / 2$, where the selection rule is exact, since also in this limit $\left\langle\left|\delta / n_{02}\right|\right\rangle$ is finite.

In Figs. 8 and 10 we show the effect of linear (blue solid curve) and quadratic (blue dots) fluctuations of $n_{02}$. The two approximations differ for small $J$, indicating that the series expansion is likely not accurate enough. This is not a problem for our description of STIRAP, which in this regime is anyway suppressed by spontaneous decay. For larger values $(J=1.32,2)$ the series expansion is seen to be accurate. *gfalci@dmfci.unict.it

${ }^{\dagger}$ Also at Department of Physics, Lancaster University, Lancaster LA1 4YB, United Kingdom.

${ }^{1}$ K. Bergmann, H. Theuer, and B. W. Shore, Rev. Mod. Phys. 70, 1003 (1998), and references therein.

${ }^{2}$ N. V. Vitanov, T. Halfmann, B. W. Shore, and K. Bergmann, Annu. Rev. Phys. Chem. 52, 763 (2001).

${ }^{3}$ E. Arimondo, in Progress in Optics, Vol. XXXV, edited by E. Wolf (North Holland, Amsterdam, 1976), pp. 257-354.

${ }^{4}$ M. O. Scully and M. S. Zubairy, Quantum Optics (Cambridge University Press, Cambridge, 1997).

${ }^{5}$ K. V. R. M. Murali, Z. Dutton, W. D. Oliver, D. S. Crankshaw, and T. P. Orlando, Phys. Rev. Lett. 93, 087003 (2004); Z. Dutton, K. V. R. M. Murali, W. D. Oliver, and T. P. Orlando, Phys. Rev. B 73, 104516 (2006).

${ }^{6}$ J. Siewert and T. Brandes, Adv. Solid State Phys. 44, 181 (2004).

${ }^{7}$ Y.-X. Liu, J. Q. You, L. F. Wei, C. P. Sun, and F. Nori, Phys. Rev. Lett. 95, 087001 (2005).

${ }^{8}$ J. Siewert, T. Brandes, and G. Falci, Opt. Commun. 264, 435 (2006).

${ }^{9}$ J. Siewert, T. Brandes, and G. Falci, Phys. Rev. B 79, 024504 (2009).

${ }^{10}$ L. F. Wei, J. R Johansson, L. X. Cen, S. Ashhab, and F. Nori, Phys. Rev. Lett. 100, 113601 (2008).

${ }^{11}$ Jian Li, G. S. Paraoanu, Katarina Cicak, Fabio Altomare, Jae I. Park, Raymond W. Simmonds, Mika A. Sillanpää, and Pertti J. Hakonen, Sci. Rep. 2, 645 (2012).

${ }^{12}$ M. H. S. Amin, A. Yu. Smirnov, and A. Maassen van den Brink, Phys. Rev. B 67, 100508 (2003); Z. Zhou, Shih-I Chu, and S. Han, ibid. 70, 094513 (2004).

${ }^{13}$ M. Mariantoni, M. J. Storcz, F. K. Wilhelm, W. D. Oliver, A. Emmert, A. Marx, R. Gross, H. Christ, and E. Solano, arXiv:cond-mat/0509737.

${ }^{14}$ J. Q. You and F. Nori, Nature (London) 474, 589 (2011), and references therein.

${ }^{15}$ M. A. Sillanpää, J. Li, K. Cicak, F. Altomare, J. I. Park, R. W. Simmonds, G. S. Paraoanu, and P. J. Hakonen, Phys. Rev. Lett. 103, 193601 (2009).

${ }^{16}$ M. Baur, S. Filipp, R. Bianchetti, J. M. Fink, M. Göppl, L. Steffen, P. J. Leek, A. Blais, and A. Wallraff, Phys. Rev. Lett. 102, 243602 (2009).

${ }^{17}$ A. A. Abdumalikov, O. Astafiev, A. M. Zagoskin, Yu. A. Pashkin, Y. Nakamura, and J. S. Tsai, Phys. Rev. Lett. 104, 193601 (2010); P. M. Anisimov, J. P. Dowling, and B. C. Sanders, ibid. 107, 163604 (2011).
${ }^{18}$ R. Bianchetti, S. Filipp, M. Baur, J. M. Fink, C. Lang, L. Steffen, M. Boissonneault, A. Blais, and A. Wallraff, Phys. Rev. Lett. 105, 223601 (2010).

${ }^{19}$ W. R. Kelly, Z. Dutton, J. Schlafer, B. Mookerji, T. A. Ohki, J. S. Kline, and D. P. Pappas, Phys. Rev. Lett. 104, 163601 (2010)

${ }^{20}$ J. Clarke and F. K. Wilhelm, Nature (London) 453, 1031 (2008).

${ }^{21}$ V. Bouchiat, D. Vion, P. Joyez, D. Esteve, and M. H. Devoret, Phys. Scr. T 76, 165 (1998).

${ }^{22}$ Y. Nakamura, Yu. Pashkin, and J. S. Tsai, Nature (London) 398, 786 (1999).

${ }^{23}$ D. Vion, A. Aassime, A. Cottet, P. Joyez, H. Pothier, C. Urbina, D. Esteve, and M. H. Devoret, Science 296, 886 (2002).

${ }^{24}$ A. Wallraff, D. I. Schuster, A. Blais, L. Frunzio, R. S. Huang, J. Majer, S. Kumar, S. M. Girvin, and R. J. Schoelkopf, Nature (London) 431, 162 (2004).

${ }^{25}$ T. Duty, D. Gunnarsson, K. Bladh, and P. Delsing, Phys. Rev. B 69, 140503 (2004).

${ }^{26}$ J. Koch, T. M. Yu, J. Gambetta, A. A. Houck, D. I. Schuster, J. Majer, A. Blais, M. H. Devoret, S. M. Girvin, and R. J. Schoelkopf, Phys. Rev. A 76, 042319 (2007); J. A. Schreier, A. A. Houck, J. Koch, D. I. Schuster, B. R. Johnson, J. M. Chow, J. M. Gambetta, J. Majer, L. Frunzio, M. H. Devoret, S. M. Girvin, and R. J. Schoelkopf, Phys. Rev. B 77, 180502 (2008).

${ }^{27}$ G. Falci, A. D’Arrigo, A. Mastellone, and E. Paladino, Phys. Rev. Lett. 94, 167002 (2005); E. Paladino, A. D’Arrigo, A. Mastellone, and G. Falci, Phys. Scr. T 137, 014017 (2009).

${ }^{28} \mathrm{G}$. Falci and R. Fazio, in Quantum Computer, Algorithms and Chaos, edited by G. Casati, D. L. Shepeliansky, P. Zoller, and G. Benenti (IOS, Amsterdam, 2005), pp. 363-416.

${ }^{29}$ Y. Nakamura, Yu. A. Pashkin, T. Yamamoto, and J. S. Tsai, Phys. Rev. Lett. 88, 047901 (2002); O. Astafiev, Yu. A. Pashkin, Y. Nakamura, T. Yamamoto, and J. S. Tsai, ibid. 93, 267007 (2004); E. Paladino, M. Sassetti, G. Falci, and U. Weiss, Phys. Rev. B 77, 041303 (2008).

${ }^{30}$ G. Ithier, E. Collin, P. Joyez, P. J. Meeson, D. Vion, D. Esteve, F. Chiarello, A. Shnirman, Y. Makhlin, J. Schriefl, and G. Schön, Phys. Rev. B 72, 134519 (2005).

${ }^{31}$ J. Bylander, S. Gustavsson, F. Yan, F. Yoshihara, K. Harrabi, G. Fitch, D. G. Cory, Y. Nakamura, J.-S. Tsai, and W. D. Oliver, Nat. Phys. 7, 565 (2011).

${ }^{32}$ F. Chiarello, E. Paladino, M. G. Castellano, C. Cosmelli, A. D'Arrigo, G. Torrioli, and G. Falci, New J. Phys. 14, 023031 (2012).

${ }^{33}$ D. Sank, R. Barends, R. C. Bialczak, Yu Chen, J. Kelly, M. Lenander, E. Lucero, M. Mariantoni, A. Megrant, M. Neeley, 
P. J. J. O'Malley, A. Vainsencher, H. Wang, J. Wenner, T. C. White, T. Yamamoto, Y. Yin, A. N. Cleland, and J. M. Martinis, Phys. Rev. Lett. 109, 067001 (2012).

${ }^{34}$ N. V. Vitanov, M. Fleischhauer, B. W. Shore, and K. Bergmann, Adv. At. Mol. Opt. Phys. 46, 55 (2001).

${ }^{35} \mathrm{~N}$. Timoney, I. Baumgart, M. Johanning, A. F. Varon, M. B. Plenio, A. Retzker, and Ch. Wunderlich, Nature (London) 476, 185 (2011).

${ }^{36}$ L. P. Yatsenko, V. I. Romanenko, B. W. Shore, and K. Bergmann, Phys. Rev. A 65, 043409 (2002).

${ }^{37}$ G. Falci, M. Berritta, A. Russo, A. D’Arrigo, and E. Paladino, Phys. Scr. T 151, 014020 (2012).

${ }^{38}$ E. Collin, G. Ithier, A. Aassime, P. Joyez, D. Vion, and D. Esteve, Phys. Rev. Lett. 93, 157005 (2004).

${ }^{39}$ G. Mangano, J. Siewert, and G. Falci, Eur. Phys. J. Special Topics 160, 259 (2008).

${ }^{40}$ C. Rigetti, J. M. Gambetta, S. Poletto, B. L. T. Plourde, J. M. Chow, A. D. Córcoles, J. A. Smolin, S. T. Merkel, J. R. Rozen, G. A. Keefe, M. B. Rothwell, M. B. Ketchen, and M. Steffen, Phys. Rev. B 86, 100506 (2012).

${ }^{41}$ E. Paladino, L. Faoro, G. Falci, and R. Fazio, Phys. Rev. Lett. 88, 228304 (2002); E. Paladino, L. Faoro, and G. Falci, Adv. Solid State Phys. 43, 747 (2003); Y. M. Galperin, B. L. Altshuler, J. Bergli, and D. V. Shantsev, Phys. Rev. Lett. 96, 097009 (2006); J. Bergli, Y. M. Galperin, and B. L. Altshuler, New J. Phys. 11, 025002 (2009); E. Paladino et al., Chem. Phys. 296, 325 (2004).

${ }^{42}$ A. B. Zorin, F. J. Ahlers, J. Niemeyer, T. Weimann, H. Wolf, V. A. Krupenin, and S. V. Lotkhov, Phys. Rev. B 53, 13682 (1996); S. Kafanov, H. Brenning, T. Duty, and P. Delsing, ibid. 78, 125411 (2008).

${ }^{43}$ U. Weiss, Quantum Dissipative Systems (World Scientific, Singapore, 1993).

${ }^{44}$ E. Paladino, A. Mastellone, A. D’ Arrigo, and G. Falci, Phys. Rev. B 81, 052502 (2010); E. Paladino, A. D'Arrigo, A. Mastellone, and G. Falci, New J. Phys. 13, 093037 (2011); B. Bellomo,
G. Compagno, A. D’ Arrigo, G. Falci, R. Lo Franco, and E. Paladino, Phys. Rev. A 81, 062309 (2010); A. D'Arrigo and E. Paladino, New J. Phys. 14, 053035 (2012).

${ }^{45} \mathrm{D}$. Vion (private communication).

${ }^{46}$ A. La Cognata, P. Caldara, D. Valenti, B. Spagnolo, A. D. Arrigo, E. Paladino, and G. Falci, Int. J. Quantum. Inform. 9, 1 (2011).

${ }^{47}$ A. Kuhn, M. Hennrich, T. Bondo, and G. Rempe, Appl. Phys. B 69, 373 (1999).

${ }^{48}$ E. Geva, R. Koslov, and R. Skinner, J. Chem. Phys. 102, 8541 (1995); Q. Shi and E. Geva, ibid. 119, 11773 (2003).

${ }^{49}$ P. A. Ivanov, N. V. Vitanov, and K. Bergmann, Phys. Rev. A 70, 063409 (2004).

${ }^{50}$ L. Viola and S. Lloyd, Phys. Rev. A 58, 2733 (1998); L. Faoro and L. Viola, Phys. Rev. Lett. 92, 117905 (2004); G. Falci, A. D’Arrigo, A. Mastellone, and E. Paladino, Phys. Rev. A 70, 040101 (2004).

${ }^{51}$ P. Facchi, S. Tasaki, S. Pascazio, H. Nakazato, A. Tokuse, and D. A. Lidar, Phys. Rev. A 71, 022302 (2005).

${ }^{52}$ I. Chiorescu, Y. Nakamura, C. J. P. M. Harmans, and J. E. Mooij, Science 299, 1869 (2003); T. Yamamoto, Yu. A. Pashkin, O. Astafiev, Y. Nakamura, and J. S. Tsai, Nature (London) 425, 941 (2003); J. B. Majer, F. G. Paauw, A. C. J. ter Haar, C. J. P. M. Harmans, and J. E. Mooij, Phys. Rev. Lett. 94, 090501 (2005); I. Chiorescu, P. Bertet, K. Semba, Y. Nakamura, C. J. P. M. Harmans, and J. E. Mooij, Nature (London) 431, 159 (2004).

${ }^{53}$ J. M. Martinis, S. Nam, J. Aumentado, and C. Urbina, Phys. Rev. Lett. 89, 117901 (2002); S. Poletto, F. Chiarello, M. G. Castellano, J. Lisenfeld, A. Lukashenko, C. Cosmelli, G. Torrioli, P. Carelli, and A. V. Ustinov, New J. Phys. 11, 013009 (2009).

${ }^{54}$ J. M. Martinis, S. Nam, J. Aumentado, K. M. Lang, and C. Urbina, Phys. Rev. B 67, 094510 (2003).

${ }^{55}$ N. Vogt, J. H. Cole, M. Marthaler, and G. Schön, Phys. Rev. B 85, 174515 (2012).

${ }^{56}$ E. Paladino, A. D’Arrigo, and G. Falci, Phys. Scr. T 151, 014048 (2012). 